\title{
Rapid and complete response to idelalisib in a case of Richter syndrome
}

This article was published in the following Dove Medical Press journal: OncoTargets and Therapy

\section{Cristina Bagacean ${ }^{1-3}$ \\ Mihnea Zdrenghea ${ }^{4}$ \\ Hussam Saad' \\ Christian Berthou ${ }^{1,2}$ \\ Yves Renaudineau ${ }^{3}$ \\ Adrian Tempescul ${ }^{1,2}$}

'Department of Hematology, Brest University Medical School Hospital, Brest, France; ${ }^{2} \mathrm{U} / 227$ B Lymphocytes and Autoimmunity, University of Brest, INSERM, IBSAM, Brest, France; ${ }^{3}$ Laboratory of Immunology and Immunotherapy, Brest University Medical School Hospital, Brest, France; ${ }^{4}$ Iuliu Hatieganu University of Medicine and Pharmacy, Cluj-Napoca, Romania
Correspondence: Cristina Bagacean Department of Hematology, Brest University Medical School Hospital, 2 Avenue Foch, Brest 29609, France

$\mathrm{Tel}+33298223421$

Fax +33 298223835

Email cristina.bagacean@univ-brest.fr

\begin{abstract}
Richter syndrome (RS) is an aggressive lymphoma arising on the back of chronic lymphocytic leukemia (CLL)/small lymphocytic lymphoma (SLL) and is the most common B-cell malignancy in the Western world. In the majority of cases, RS presents an activated $B$ cell (ABC) phenotype of diffuse large B-cell lymphoma (DLBCL). From the therapeutic point of view, selective inhibition of $\mathrm{PI} 3 \mathrm{~K} \delta$ with idelalisib represents a valuable addition to available treatment options for patients with CLL/SLL, many of whom do not respond to or cannot tolerate chemoimmunotherapy. However, to our knowledge, there have been no prospective studies evaluating idelalisib efficacy in a DLBCL-ABC form of RS. Here, we present a case of a DLBCL-ABC form of RS achieving a complete response at 3 weeks after initiating idelalisib and rituximab therapy for six cycles. This response was maintained during the idelalisib monotherapy, but the patient relapsed rapidly after treatment was withdrawn, because of a grade three immune colitis that developed at 10 months of treatment. This report demonstrates that idelalisib is highly effective in RS and provides an attractive option in this aggressive disease. This agent could meet an unmet need by providing a treatment option with a tolerable safety profile for elderly patients with RS.
\end{abstract}

Keywords: chronic lymphocytic leukemia, small lymphocytic lymphoma, Richter syndrome, idelalisib

\section{Introduction}

Chronic lymphocytic leukemia (CLL) is the most common B-cell malignancy in the Western world, with a median age at diagnosis of 72 years. Small lymphocytic lymphoma (SLL) is a mature B-cell neoplasm considered identical to CLL, but this term is used when involvement is primarily nodal. Richter syndrome (RS) is an aggressive lymphoma arising on the back of known CLL/SLL. ${ }^{1}$ The vast majority of patients with RS transform from CLL/SLL to an activated B-cell (ABC) phenotype of diffuse large B cell lymphoma (DLBCL), with a small minority transforming to classic Hodgkin's lymphoma or other non-Hodgkin's lymphomas, including Burkitt's lymphoma and lymphoblastic lymphoma. ${ }^{2,3}$ Typically, RS has an aggressive presentation, chemotherapy resistance, and very poor survival. Europe is currently the world's major area with the highest proportion of older persons in the population, and it is projected to remain so for at least the next 50 years. As the proportion of older persons relative to the rest of the population has increased considerably, treatment of CLL/SLL and RS has become an important public health problem. Optimal therapeutic management in both CLL/SLL and RS patients requires a choice of treatment adapted to avoid premature mortality due to treatment toxicity, and above all promoting a beneficial effect on overall survival and quality of life. 
The B-cell receptor signaling pathway plays a critical role in the pathogenesis of CLL/SLL and RS. Recently approved inhibitors of the B-cell-receptor signaling pathway, including the PI3K $\delta$ inhibitor idelalisib, have significantly improved the outcomes of relapsed and refractory CLL/ SLL patients. ${ }^{46}$ When administered to patients with CLL/ SLL with extensive prior treatment, idelalisib shows durable antitumor activity accompanied by sustained or improved quality-of-life outcomes. Idelalisib has an acceptable safety profile, confirmed by a pivotal Phase III randomized trial of idelalisib plus rituximab vs rituximab plus placebo. ${ }^{6}$ However, in some cases, serious or fatal diarrhea/colitis, hepatotoxicity, pneumonitis, and intestinal perforation have been reported. In comparison to other treatment modalities, idelalisib is effective, regardless of the $17 \mathrm{p}$-deletion status or $I G H V$-mutation status. ${ }^{7}$ In Europe and the US, idelalisib is approved in combination with rituximab or ofatumumab for the treatment of patients with relapsed CLL/SLL as first-line therapy in CLL patients with $17 \mathrm{p}$ chromosomal deletion or TP53 mutation considered unsuitable for chemoimmunotherapy and as monotherapy for patients with refractory follicular lymphoma. ${ }^{7}$ However, no data are available yet from prospective studies testing the efficacy of idelalisib in RS or DLBCL.

\section{Case report}

A 66-year-old Caucasian man was initially referred to us from a hematological consultation for enlarged cervical and axillary lymph nodes. A cervical lymph-node biopsy was performed, revealing SLL with a normal karyotype. No circulating clonal cells were revealed by flow cytometry in the peripheral blood, but $16 \%$ infiltration with clonal cells was revealed in a bone-marrow biopsy. After 2 years of follow-up, due to progressive lymphadenopathies and the presence of a bulky abdominal mass, a new biopsy was performed, which was still suggestive of SLL. The patient did not present any B-type constitutional symptoms. The patient was treated with three cycles of cyclophosphamide-doxorubicin-vincristineprednisone and three cycles of rituximab-fludarabine, and a complete response (CR) was obtained.

After 11 years of remission, at the age of 77 years, the patient consulted his hematologist, because lymphadenopathies had been observed in the cervical region. Indeed, clinical examination revealed left submandibular and left preauricular adenopathies. Fine-needle aspiration was performed, which confirmed the relapse of the low-grade SLL. Initially, it was decided to take a watch-and-wait approach, but after only 1 month, the cervical adenopathies had doubled in volume and the preauricular mass was generating significant pain.
Computed tomography was performed, showing a large left cervical lymph-node conglomerate extending from the upper jugulocarotid territory to the left supraclavicular territory and mediastinum, sheathing the jugulocarotid vascular system $\left(75 \times 35 \mathrm{~mm}, 2,669 \mathrm{~mm}^{2}\right)$, lymph-node invasion of the left parotid lodge $\left(43 \times 23 \mathrm{~mm}, 1,030 \mathrm{~mm}^{2}\right)$, multiple left supraclavicular adenopathies $\left(29 \times 15 \mathrm{~mm}, 450 \mathrm{~mm}^{2}\right)$, right retrotracheal mediastinal lymphadenopathy, and no visceral involvement (Figure 1A).

Blood tests revealed a normal complete blood count, no circulating clonal cells were identified by flow-cytometry analysis, and LDH levels, reflecting tumor mass, were slightly elevated, at $398 \mathrm{U} / \mathrm{L}$ (normal values 208-378 U/L). A new biopsy of a cervical lymph node was performed, revealing a DLBCL with an $\mathrm{ABC}$ phenotype $\left(\mathrm{CD}^{2} 5^{+}\right.$, $\mathrm{CD} 20^{+}, \mathrm{BCL}^{2+}, \mathrm{CD} 10^{-}, \mathrm{BCL6}^{-}$) and a $\mathrm{Ki} 67$ index of proliferation of $40 \%$ (Figure 2). Cytogenetic analysis of the lymph node revealed an abnormal clone with a $10 \mathrm{p}$ deletion (46,XY,del[10][p11][6]/46,XY[12]). Accordingly, the patient was diagnosed with a DLBCL-ABC type variant of RS, stage IIA (Ann Arbor).

Treatment with rituximab and idelalisib $150 \mathrm{mg}$ twice a day was initiated based on the information regarding SLL relapse, but before having the DLBCL-ABC form of RS histopathology results. Fluorodeoxyglucose positronemission tomography (FDG-PET) was performed 3 weeks after treatment initiation to have an adapted evaluation of the extension of the DLBCL. Surprisingly, this revealed the absence of lymph-node hypermetabolism in the cervical region that was associated with the presence of several inflammatory hypermetabolic mediastinal lymph nodes, supporting a CR after only 3 weeks of rituximab-idelalisib treatment (Figure 1B). CR was maintained and confirmed on FDG-PET performed at 3 months and 6 months after treatment initiation (Figure 1C and D). After six cycles of rituximab-idelalisib, idelalisib was continued as a single agent for 10 months when the patient developed grade three idelalisib-related immune colitis, necessitating treatment discontinuation. Stool specimens were collected for routine bacterial and parasitological testing and stool toxins for Clostridium difficile, which were negative. After treatment discontinuation, the diarrhea resolved, but after only 1 month, cervical adenopathies had reappeared at the clinical examination. FDG-PET confirmed the relapse of the DLBCL, and rituximab-PMitCEBO (prednisolone-mitoxantronecyclophosphamide-etoposide-bleomycin-vincristine) treatment was initiated. The patient is currently on his first immunochemotherapy cycle. 


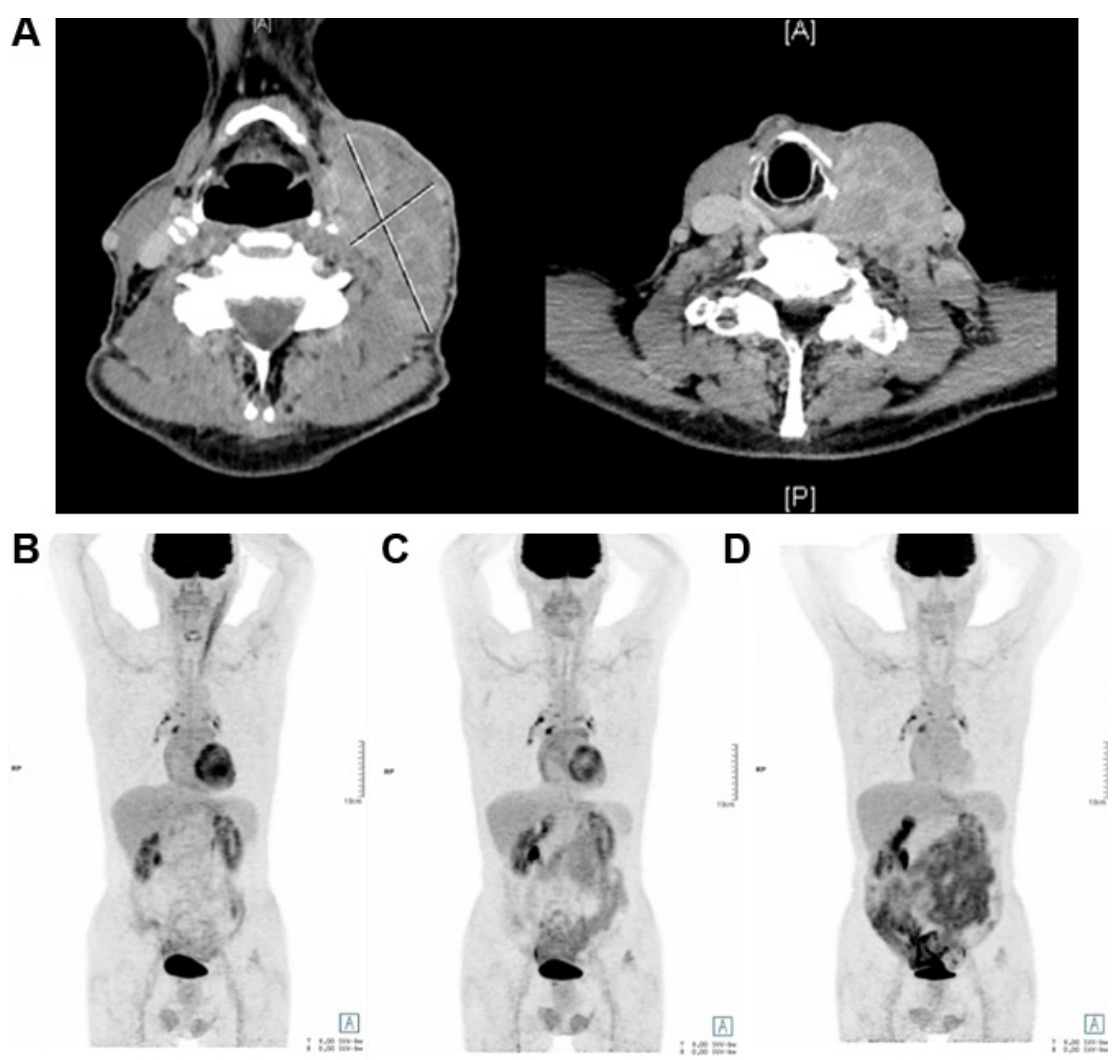

Figure I Computed tomography (CT) and fluorodeoxyglucose positron-emission tomography (FDG-PET) scans.

Notes: (A) CT performed before rituximab-idelalisib treatment initiation, showing a large left cervical lymph node conglomerate extending from upper jugulocarotid territory to left supraclavicular territory, sheathing the jugulocarotid vascular system, lymph-node invasion of the left parotid lodge, and multiple left supraclavicular adenopathies. (B) FDG-PET performed after 3 weeks of rituximab-idelalisib treatment, revealing the absence of lymph-node hypermetabolism in the cervical region associated with the presence of several inflammatory hypermetabolic mediastinal lymph nodes supporting a complete response. Complete response was maintained and confirmed by FDG-PET performed at 3 months (C) and 6 months (D) after treatment initiation.

\section{Discussion and conclusion}

We here report the case of a patient with a DLBC-ABC variant of RS achieving a CR to the PI3K $\delta$ inhibitor idelalisib when associated with rituximab for six cycles. The report demonstrates that the combination of rituximab-idelalisib is highly effective in RS, as CR was rapidly achieved after only 3 weeks of treatment. We also report that the patient relapsed soon after treatment was discontinued. Among
A
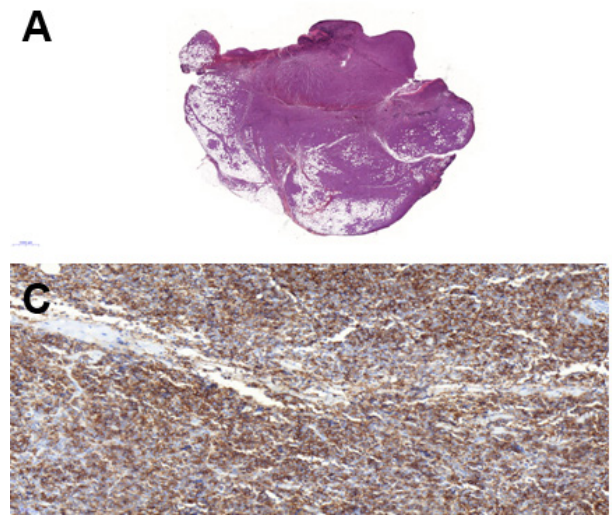
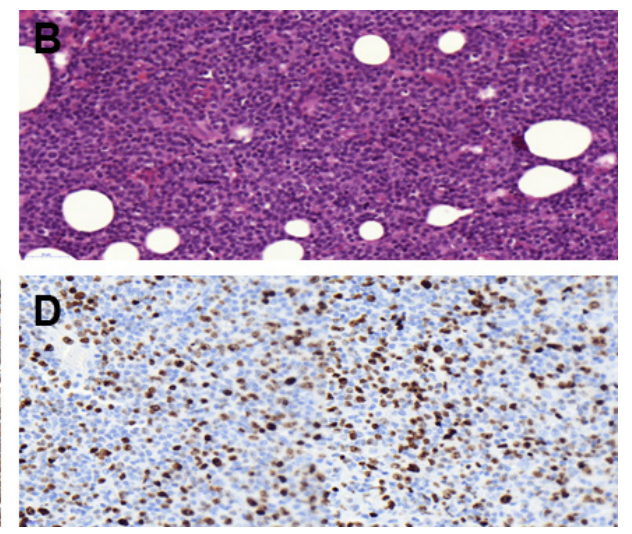

Figure 2 Histological examination of a cervical lymph node, revealing the transformation of SLL into a DLBCL with an ABC phenotype.

Notes: (A) HES staining, OM I.I $\times$, showing lymph node and periganglionic infiltration. (B) HES staining, OM 39.I $\times$, showing infiltration with large cells with a large nucleus, containing a large nucleolus and some images of mitosis. (C) CD20 immunostaining, OM 19.6×. (D) Ki67 immunostaining, OM 40.7×, showing an index of proliferation at 40\%. Abbreviations: SLL, small lymphocytic lymphoma; DLBCL, diffuse large B-cell lymphoma; ABC, activated B cell; HES, hematoxylin-eosin-saffron; OM, original magnification. 
the different mechanisms that could explain this relapse, Georgios et al have reported that blocking the $\mathrm{PI} 3 \mathrm{~K} \delta$ isoform in DLBCL-ABC cell lines was counterbalanced by activation of the PI3K $\alpha$ isoform, which generated treatment resistance. ${ }^{8}$

To our knowledge, there was only a small case series recently from Visentin et al on four subjects with a DLBCL variant of RS, which were treated with idelalisib as monotherapy. ${ }^{9}$ In this case series, one patient achieved $\mathrm{CR}$, two patients partial response, and one patient stable disease. None of these patients progressed during a median follow-up period of 5.1 \pm 2.3 months. A larger cohort and longer follow-up are needed, but together with our report, these data provide encouraging evidence that idelalisib is active in the DLBCL-ABC form of RS. Although the toxicity profile associated with $\mathrm{PI} 3 \mathrm{~K}$ inhibitors, in particular the gastrointestinal toxicity and the high risk of infection, may have curbed enthusiasm for these agents, idelalisib could fulfill an unmet need by providing a treatment option for elderly patients with RS.

\section{Consent for publication}

Written informed consent was provided by the patient to have the case details and any accompanying images published. Institutional approval from the University Hospital Brest Ethical Committee was obtained to publish the case details. The patient is included in the cohort OFICE (ClinicalTrials. gov: NCT03294980).

\section{Acknowledgment}

The authors express their gratitude to Simone Forest and Geneviève Michel for their secretarial help and to Professor Wesley Brooks for proofreading and editing the manuscript.

\section{Disclosure}

The authors report no conflicts of interest in this work.

\section{References}

1. Swerdlow SH, Campo E, Pileri SA, et al. The 2016 revision of the World Health organization classification of lymphoid neoplasms. Blood. 2016;127(20):2375-2390.

2. Bockorny B, Codreanu I, Dasanu CA. Hodgkin lymphoma as Richter transformation in chronic lymphocytic leukaemia: a retrospective analysis of world literature. Br J Haematol. 2012;156(1):50-66.

3. Eyre TA, Schuh A. An update for Richter syndrome - new directions and developments. Br J Haematol. 2017;178(4):508-520.

4. Brown JR, Byrd JC, Coutre SE, et al. Idelalisib, an inhibitor of phosphatidylinositol 3-kinase $\mathrm{p} 110 \delta$, for relapsed/refractory chronic lymphocytic leukemia. Blood. 2014;123(22):3390-3397.

5. Byrd JC, Brown JR, O'Brien S, et al. Ibrutinib versus of atumumab in previously treated chronic lymphoid leukemia. N Engl J Med. 2014;371(3): 213-223.

6. Furman RR, Sharman JP, Coutre SE, et al. Idelalisib and rituximab in relapsed chronic lymphocytic leukemia. N Engl J Med. 2014;370(11): 997-1007.

7. Puła A, Stawiski K, Braun M, Iskierka-Jażdżewska E, Robak T. Efficacy and safety of B-cell receptor signaling pathway inhibitors in relapsed/refractory chronic lymphocytic leukemia: a systematic review and meta-analysis of randomized clinical trials. Leuk Lymphoma. 2018;59(5):1084-1094.

8. Pongas GN, Annunziata CM, Staudt LM. PI3K $\delta$ inhibition causes feedback activation of PI $3 \mathrm{~K} \alpha$ in the ABC subtype of diffuse large B-cell lymphoma. Oncotarget. 2017;8(47):81794-81802.

9. Visentin A, Imbergamo S, Scomazzon E, et al. BCR kinase inhibitors, idelalisib and ibrutinib, are active and effective in Richter syndrome. Br J Haematol. 2018:17.
OncoTargets and Therapy

\section{Publish your work in this journal}

OncoTargets and Therapy is an international, peer-reviewed, open access journal focusing on the pathological basis of all cancers, potential targets for therapy and treatment protocols employed to improve the management of cancer patients. The journal also focuses on the impact of management programs and new therapeutic agents and protocols on

\section{Dovepress}

patient perspectives such as quality of life, adherence and satisfaction. The manuscript management system is completely online and includes a very quick and fair peer-review system, which is all easy to use. Visit http://www.dovepress.com/testimonials.php to read real quotes from published authors. 